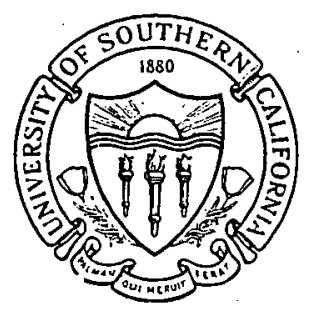

\title{
UNIVERSITY OF SOUTHERN CALIFORNIA
}

\author{
SCHOOL OF ENGINEERING
}

CONSTANT DIRECTIONS OF THE

\author{
RICCATI EQUATION
}

David Rappaport

United States Air Force Office of Scientific Research Grant No. AF-AFOSR-1244-67 and National Aeronautics and Space Administration Grant NGL-05-018-044, Supp1. \#3.

\section{DEPARTMENT OF AEROSPACE ENGINEERING}

1. This document has been approved for public release and sale; its distribution is unlimited.

(N ASA $-C R-127035)$

THE RICCATI

(University OQUATION

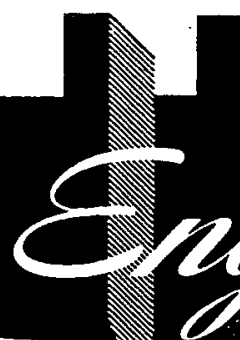

$35 \mathrm{p}$

DIRECTIONS OF

of Southern Rappaport
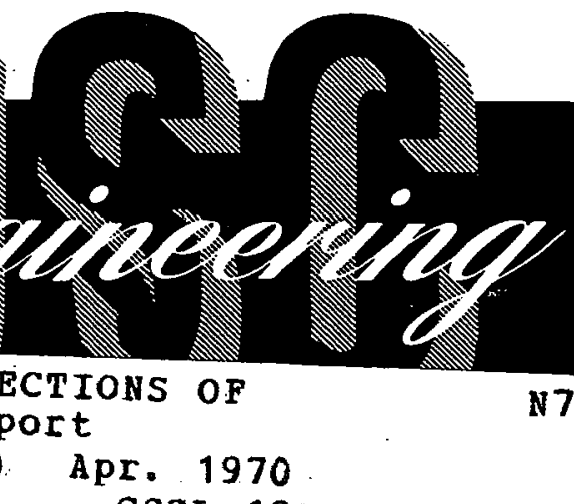

CSCL $12 \mathrm{~A}$

N72-26479 
Qualified requestors may obtain additional copies from the Defense Documentation Center, all others should apply to the Clearinghouse for Federal Scientific and Technical Information. 


\section{A CKNOW LEDGEMENTS}

The author is very grateful for the inspiration and guidance provided by Professor R. S. Bucy. His gratitude likewise extends to Professor L. M. Silverman whose suggestions and encouragement proved to be of great value.

This report was made possible by the support of the Air Force Office of Scientific Research under grant AF-AFOSR-1244-67 and NASA grant NGL-05-018-044, suppl. \#3. 


\section{NOTATION CONVENTION}

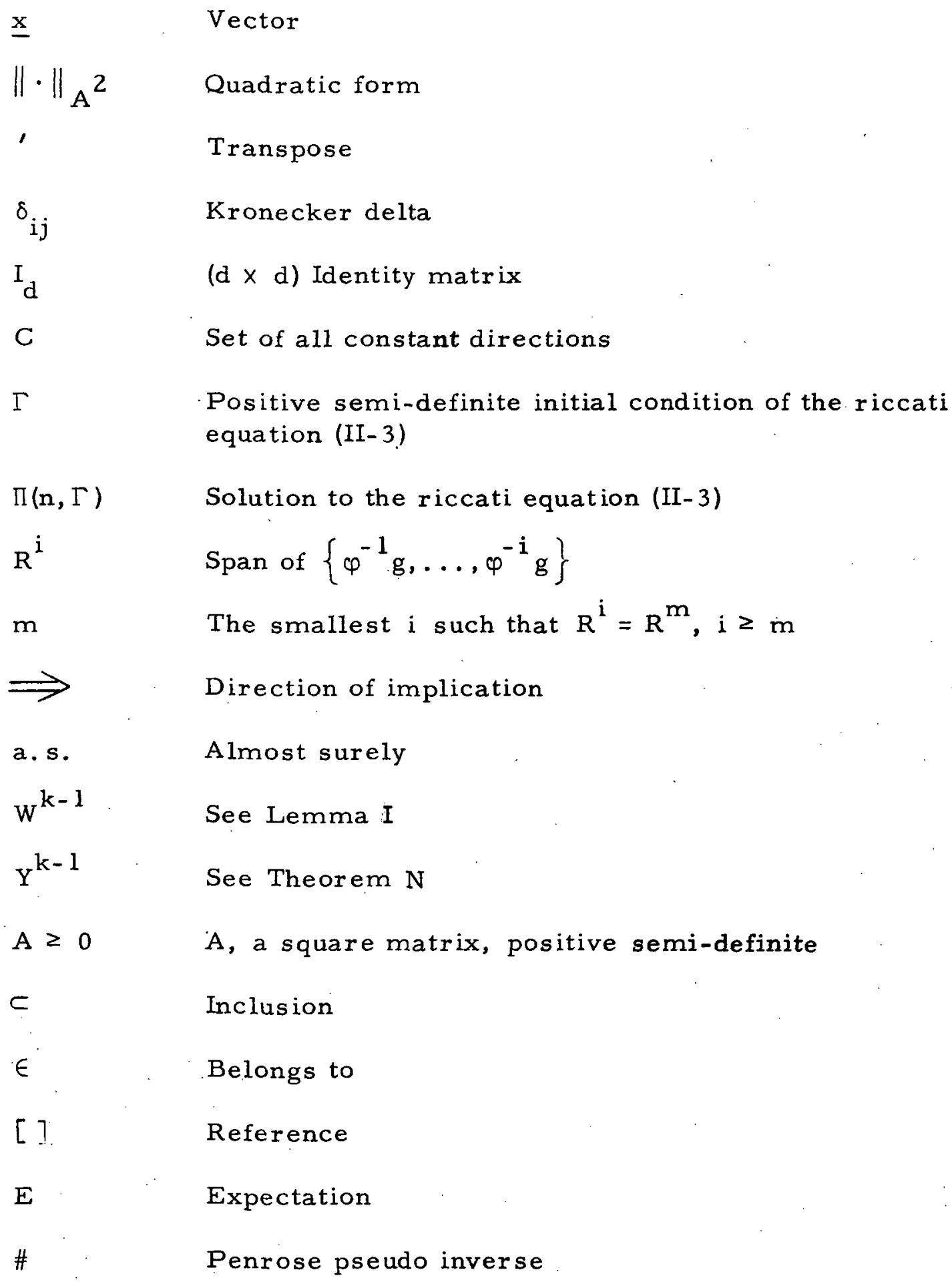




\section{CONTENTS}

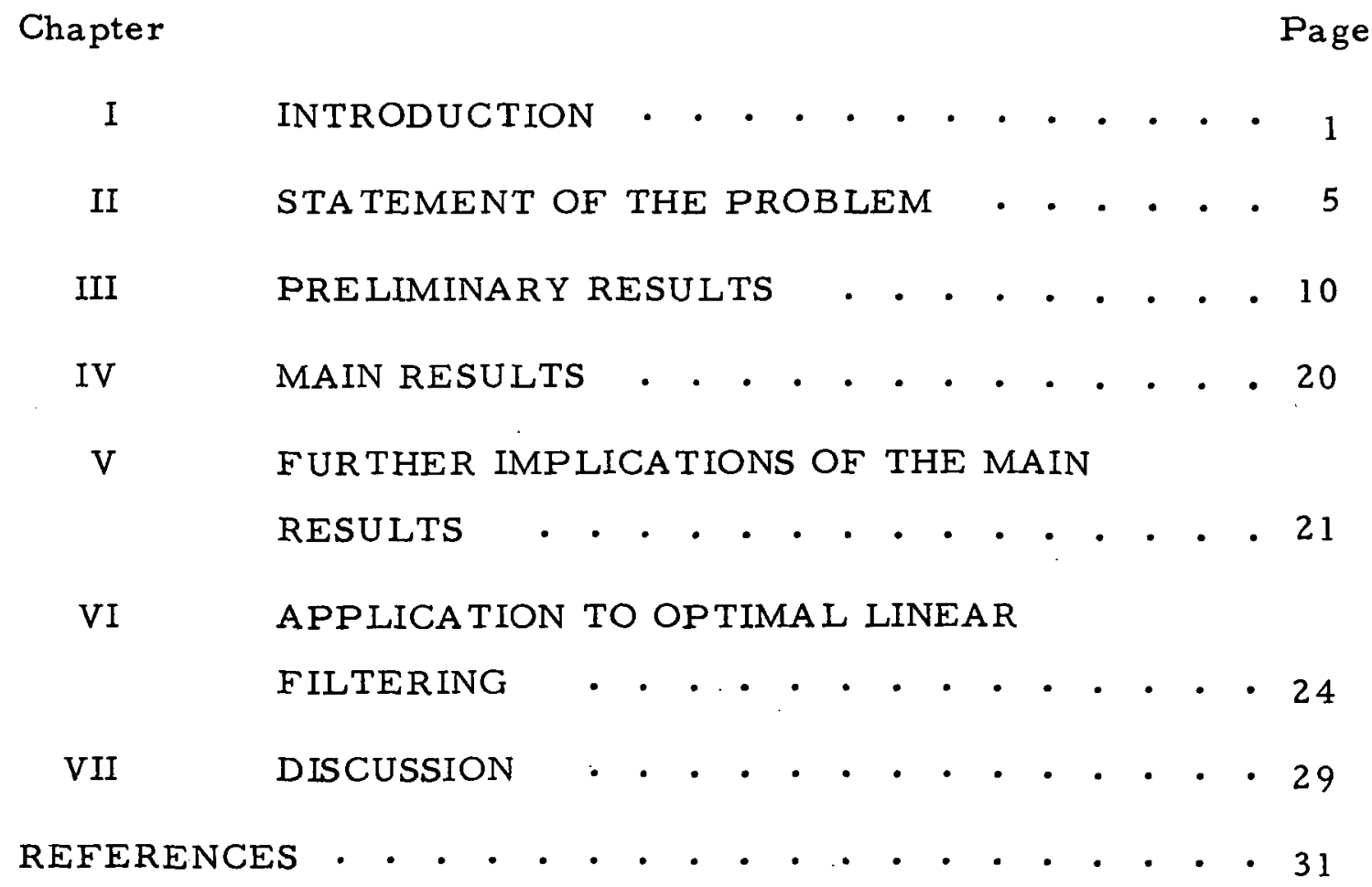




\section{CHAPTER 1}

\section{INTRODU CTION}

The appearance of riccati type equations in the solution of optimal linear quadratic control problems and in optimal linear filtering problems with gaussian randomness has been noted and studied by many researchers. In such problems the matrix of quadratic form for performance index under optimal control, and the optimal error covariance matrix, in the control and filtering situations respectively satisfy nonlinear difference equations in discrete time, nonlinear differential equations in continuous time which are of the riccati type. In references [5], [9]-[12], these equations are studied and their importance recognized in the mechanization of the optimal feedback control and the optimal filter, respectively, in the control and filtering contexts. For the particular class of problems corresponding to the cost-free-control quadratic regulator problem, in which the cost is not explicitly dependent on control, and the filtering problem in which the observations contain additive correlated noise, commonly called colored noise, it has been noted that it is possible to reduce the computational complexity of the optimal system; that is, reduction in the dimension of the optimal controller or filter. The discrete time cost-freecontrol quadratic regulator problem is discussed in reference [ 12] . The formulation and solution to the discrete time filtering problem with additive correlated observation noise is presented in reference [9] . In [9] the states of the system being observed a re totally augmented by the dynamic structure of the observation noise. 
Efforts to reduce the computational complexity of these dual control and filtering problems fall into two groups; the continuous time and the discrete time cases. In the former case the three noteworthy attempts are found in references [2], [3] and [9]. In [2] a continuous analog of the model in [9] is assumed with the states of the observed system being augmented by the correlated observation noise states. A data-differentiating algorithm is applied to the augmented model. The algorithm comprises a set of sufficient conditions for reduction in filter dimension. In [3] and [4] a particular correlated noise model is assumed and a data differencing scheme is employed which avoids the need to augment the states of the observed system. A common aspect of these two approaches is the conver sion of the given system into a different, but equivalent form, which contains white observation noise, and for which the optimal filter is derivable. The discrete time case was first considered in [6]. Another effort is made in reference [8], which is a discrete time version of [2]. Both [6] and [8] reduce the size of the totally augmented model by the number of observations, the rationale being that the totally augmented model provides perfect measurements, hence estimates in these directions are redundant. In addition [10] provides a sufficient condition for further reduction.

Undertaken in this eportis ian examination of the riccati equation arising from a linear discrete autonomous cost-free-control quadratic regulator optimal control problem with a scalar input, or equivalently by duality (see [9], [10] and [5]) that occurring in optimal filtering of a linear, discrete time autonomous, totally 
augmented system driven by a white gaussian noise sequence, observed by a noiseless scalar output. The problem is defined as a search for constant directions of the riccati equation which are independent of the initial condition. The constant directions are those directions in which the riccati equation reaches equilibrium in finite time. It is shown that the set of constant directions comprise a vector space. A set of linearly independent constant directions of dimension $K$, means a like reduction in the dimension of the nonlinear riccati equation and the elimination of constant singularities after a finite time interval. Necessary and sufficient conditions for theexistence of such constant directions are determined. These conditions comprise linear relations between the moments of the linear system. The moments are a structural feature of the system.

In the control problem a consequence of the concept "constant directions" is necessary and sufficient conditions that the dead beat control [13] be optimal for a given initial system state. For the case of filtering, the optimal one-step predictor is shown to consist of two component parts; one, corresponding to the set of constant directions, being a delay line with constant tap gains, and the other having the commonly known dynamic form utilizing the complete set of past outputs in general.

The attack is mounted via consideration of the cost-freecontrol quadratic regulator problem. While this is not necessary it provides a convenient framework in which intuition is a helpful guide. The results developed for the control case are then applied to optimal filtering by reason of duality. 
The results indicate that the discrete time data differencing techniques are not necessary to accomplish reduction in computational complexity and in actual fact do not achieve the maximum possible.

It is worthwhile to note that the discrete time cost-freecontrol quadratic regulator optimal control problem is well posed, whereas the corresponding continuous time problem is not necessarily so. As is shown in the sequel, the concept of a constant direction of the riccati equation corresponds to an optimal trajectory passing through the origin at the termination of the interval of controllability (see [13]). The analogous behavior in the case of continuous time is instantaneous removal to the origin. A condition of singularity!

This report is partitioned as follows: Chapter $I$ is an introduction. Chapter II is a detailed statement and description of the problem. Chapter III contains the derivation of preliminary results. Chapter IV consists of the main results. Chapter V is devoted to further implications of the main results. Chapter VI is the application of the results to optimal linear filtering. Chapter VII is a comparison of the results obtained here with those obtained by data-differencing. 
CHAPTER I

STA TEMENT OF THE PROBLEM

A. The cost-free-control quadratic regulator problem (see [14], Chapter 13, for more information on the discrete time optimal control problem). Consider a discrete-time optimal control problem for an autonomous linear system with a scalar input described as follows:

a) The motion of the system is specified by

$$
\begin{aligned}
& \underline{x}_{n+1}=\varphi \underline{x}_{n}+g q_{n}, n=1,2, \ldots, N \\
& \underline{x}_{0}=c
\end{aligned}
$$

where,

$$
\begin{aligned}
& \underline{x}_{n} \text { is column vector of dimension } d \\
& \varphi \text { a nonsingular }(d \times d) \text { matrix } \\
& g \text { a }(d \times 1) \text { matrix }
\end{aligned}
$$

and

$q_{n}$ a scalar, the control variable.

b) A cost not explicitly dependent on the control variable will be assigned to each trajectory on the interval $[0, \mathrm{~N}]$ by the scalar functional

$$
\mathrm{V}\left(\mathrm{N}, \Gamma ; \underline{\mathrm{c}}, 0 ; \mathrm{Q}_{0}^{\mathrm{N}}\right)=\sum_{\mathrm{i}=0}^{\mathrm{N}-1}\left\|\underline{\mathrm{x}}_{\mathrm{i}}\right\|_{\mathrm{H}^{\prime} \mathrm{H}}^{2}+\left\|\underline{\mathrm{x}}_{\mathrm{N}}\right\|_{\Gamma}^{2}
$$


where $Q_{0}^{N}$ denotes the control sequence $q_{i} i=0, \ldots, N-1$, and where

$H$ is a $(d \times r)$ matrix $d \geq r$, and

$\Gamma$ is a $(d x d)$ positive-semidefinite $(\geq 0)$ matrix.

The optimal cost is given as

$$
\begin{aligned}
& \mathrm{V}^{0}(N, \Gamma ; \underline{c} ; 0)=\min _{\mathrm{q}_{\mathrm{i}}} \mathrm{V}\left(\mathrm{N}, \Gamma ; \underline{c}, 0 ; \mathrm{Q}_{0}^{\mathrm{N}}\right)=\|\underline{c}\|_{\mathrm{P}_{\mathrm{N}}}^{2} \text {, } \\
& 0 \leq \mathrm{i} \leq \mathrm{N}-1
\end{aligned}
$$

where

$$
\begin{aligned}
& P_{N}=\varphi^{\prime} P_{N-1} \varphi-\varphi^{\prime} P_{N-1} g\left(g^{\prime} P_{N-1} g\right)^{\#} g^{\prime} P_{N-1} \varphi+H^{\prime} H \\
& P_{0}=\Gamma
\end{aligned}
$$

and \# denotes the Penrose pseudo inverse (see [12?). Denote the solution to $(I I-3)$ by $\Pi(n, \Gamma)$.

Note that the autonomous nature of the system implies that the cost is the same on any time interval of the same duration as $[0, N]$. This enables the fixing of the intital time, here conveniently taken as zero, while the final time $\mathrm{N}$ is moved ahead as optimization on intervals of increasing length are considered. This accounts for the "forward" riccati equation (II-3), rather than the usually encountered "backward" riccati equations in the optimal control setup. 
B. The correlated noise problem (see reference [9]). Consider a discrete-time optimal filtering problem for an autonomous system with a scalar output described as follows:

a) The motion of the system is specified by

$$
\begin{gathered}
\underline{x}_{n+1}=\psi \underline{x}_{n}+G u_{n} \\
\therefore \\
\underline{x}_{0}=c
\end{gathered}
$$

where,

$\underline{x}_{\mathbf{n}}$ is a d-dimensional column matrix,

$\psi$ is a $(d \times d)$ nonsingular matrix,

$G$ is a $(d x r)$ matrix, $d \geq r$,

$\underline{u}_{n}$ is a vector zero-mean gaussian white noise sequence, independent of $x_{n}$ with covariance $E_{\underline{u}_{i-j}} u_{i j}^{\prime}=\delta_{i j}{ }_{r}$.

c is a zero-mean gaussian random variable independent of $\underline{u}_{n}$, with covariance $E_{c c}^{\prime}=\Gamma$.

The scalar output of the system is given by

$$
z_{n}=h x_{n}
$$

where

$h$ is a $(1 \times d)$ matrix.

(II-4) and (II-5) are the dynamic equation for the so-called totally augmented system. 
b) As is well known $([9],[11])$ a random variable $\hat{\underline{x}}_{n+1 / n}$ which minimizes $E\left[\underline{\lambda}^{\prime}\left(\underline{x}_{n+1}-\hat{x}_{n+1 / n}\right]^{2}\right.$ for all vectors $\underline{\lambda}$, is given by the gaussian random variable which is the expected value of $\underline{x}_{n+1}$ given the observed sequence, $z_{i}, 0 \leq i \leq n$. This random variable is generated by the difference equation

$$
\underline{\hat{x}}_{n+1 / n}=\varphi \hat{x}_{n / n-1}+\varphi P_{n} h^{\prime}\left(h P_{n} h^{\prime}\right)^{\#}\left(Z_{n}-h \hat{x}_{n / n-1}\right) \text { a.s. }
$$

\# denoting the Penrose-pseudo inverse, and where $P_{n}$, the error covariance function

$$
E\left(\underline{x}_{n}-\hat{x}_{n / n-1}\right)\left(\underline{x}_{n}-\hat{x}_{n / n-1}\right)^{\prime}=P_{n}
$$

satisfies the nonlinear riccati difference equation

$$
\begin{gathered}
P_{n+1}=\psi P_{n} \psi^{\prime}-\psi P_{n} h^{\prime}\left(h P_{n} h^{\prime}\right)^{\#} h P_{n} \psi^{\prime}+G G^{\prime} \\
P_{0}=\Gamma
\end{gathered}
$$

C. The optimal control and optimal filtering problems just outlined are duals of one another under the mappings

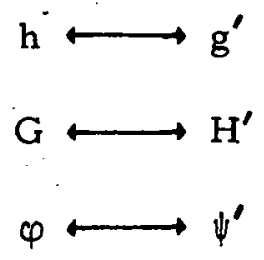


An immediate result under this duality transformation is that the riccati equations (II-3) and (II- 7) map into each other. See references [5 ], [9] and $[11]$ for more information on duality.

As is clear, the riccati equation is central to the solution of optimal linear control and linear filtering problems. An important concern in the implementation of the optimal filter and optimal controller is the dynamic dimension of the riccati equation, i. e., the number of independent varying directions. It is shown in [6] and [8] that for the colored noise problem the iteration for $P_{n}$, which is a $(d \times d)$ matrix, can essentially be reduced to iterations of matrices of size $((d-s) \times(d-s))$, $s$ being the dimension of the observation in the filtering case, that of the control in the control case. The problem to be examined in the sequel will be restricted to the case of $s=1$, although some of the preliminary lemmas which are proved are in actuality not so constrained. The problem to be confronted can be phrased as follows:

"To find the set of all linearly independent directions C for which the vector $\Pi(n, \Gamma) \underline{c}, \underline{c} \in C$, reaches an equilibrium point in finite time, for all initial conditions $\Gamma$, where $\Pi(n, \Gamma)$ is the solution of equation (II-3)." 


\section{CHAPTER II I}

\section{PRELIMINARY RESULTS}

This chapter is a compilation of definitions, theorems and lemmas needed for the derivation of the main results which are summarized in the chapter following. Definition A formulates the concept of a constant direction of the riccati equation. Theorem $B$ reveals its physical meaning. The remainder of the results of this section further characterize and describe the set of constant directions.

The control system and the corresponding riccati equation to which reference will be made in this section are described by equations $(I I-I),(I I-2)$, and $(I I-3)$.

DEFINITION 1: $\quad c$ is a constant direction of equation (II-3) if there exists some finite $N$ such that for all $n \geq N$ and each $\Gamma_{1} \geq 0$, each $\Gamma_{2} \geq 0,\|\underline{c}\|_{\Pi\left(n, \Gamma_{1}\right)}^{2}=\mid \underline{c} \|_{\Pi\left(n, \Gamma_{2}\right)}^{2}$ Let the set of al1 constant directions of $(I I-3)$ be denoted by $C$.

Note the $\Gamma$-independence of $C$.

THEOREM A: $\quad \underline{c} \varepsilon \mathrm{C}$ iff $\exists N 3 \forall n, m \geq N$ and for each $\Gamma_{1} \geq 0$, each $\Gamma_{2} \geq 0,\left\|\left.\underline{c}\right|_{\Pi\left(n, \Gamma_{1}\right)}=\right\| \underline{c} \|_{\Pi\left(m, \Gamma_{2}\right)}^{2}$.

PROOF: If : take $n=m$. Conversely if $\underline{c} \in C$, then

||$\underline{c} \|_{\Pi\left(n, \Gamma_{1}\right)}=\left.|| \underline{c}\right|_{\Pi\left(n, H^{\prime} H\right)} ^{2}=\left.|| \underline{c}\right|_{\Pi(n+1,0)} ^{2}=\left.|| \underline{c}\right|_{\Pi\left(n+1, \Gamma_{2}\right)} ^{2}$ and the theorem follows inductively. 
Theorem A provides an alternate definition of $C$.

DEFINITION 2: $\quad \underline{c} \in C_{N}, N$ finite, if for $\forall n \geq N$, each $\Gamma_{1} \geq 0$, each $\Gamma_{2} \geq 0,\|\underline{c}\|_{\Pi\left(n, \Gamma_{1}\right)}^{2}=\|\underline{c}\|_{\Pi\left(n, \Gamma_{2}\right)}^{2}$.

Note that by definitions 1 and $2, C_{N} \subset C$, that $C_{j} \subset C_{j} i \leq j$ and that if $\underline{c} \varepsilon C$, then $\underline{c} \varepsilon C_{k}$ for some finite $k \geq 1$.

Remark: In the sequel the statement " $\underline{c}$ can be taken optimally to the origin by $(I I-1),(I I-2)$ on $[0, N]$ for $\Gamma=\Gamma^{*} "$ means that for system (II-1), (II-2) with terminal cost $\Gamma^{\star}$ there exists a set of optimal controls $q_{j}, i=0, \ldots, N-1$ and a corresponding optimal trajectory $c, \underline{x}_{1}, \underline{x}_{2}, \ldots, \underline{x}_{N}$ where $\underline{x}_{N}=\underline{0}$. System $(I I-1),(I I-2)$ will henceforth be denoted by the symbol *.

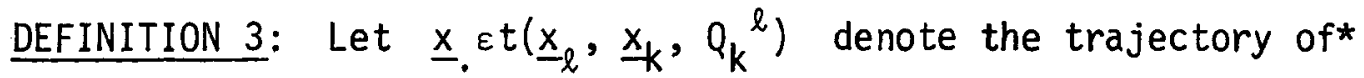
on $[k, \ell] \quad \underline{x}_{k}, \underline{x}_{k+1}, \ldots, \underline{x}_{\ell}$, generated by the control sequence $q_{k}, \ldots, q_{\ell-1}$, which is denoted by $Q_{k}^{l}$.

THEOREM B: $\underline{c} \varepsilon C_{N}$ for some finite $N$ iff $\underline{c}$ can be taken optimally to the origin by * on $[0, N]$ for $\Gamma=0$, (i.e., $\underline{x}_{N-1}=\phi^{-1} g \alpha$, some $\alpha$ ). The following two lemmas will be used in the proof of Theorem $B$. Lemma a : $V^{0}(N, 0 ; \underline{c}, 0) \leq V^{0}(n, \Gamma ; \underline{c} 0) \forall n \geq N, \forall \Gamma \geq 0$ and each $\underline{c}$.

Proof: Let $Q_{0}{ }^{n}$ denote the control sequence $q_{0}, \ldots, q_{n-1}$. Let $Q_{0}^{N}$ denote the first $N$ controls. Then for each $\Gamma \geq 0$, each $c$ and each $n \geq N \quad V\left(N, 0 ; \underline{c}, 0 ; Q_{0}^{N}\right) \leq V\left(n, \Gamma ; \underline{c}, 0 ; Q_{0}{ }^{n}\right)$ by (II-2). Then $V^{0}(N, 0 ; \underline{c}, 0) \leq V\left(n, \Gamma ; \underline{c}, 0 ; Q_{0}{ }^{n}\right)$ for any $Q_{0}{ }^{n}$. The result follows. 
Lemma b: If $\underline{c}$ can be taken optimally to the origin by * for $\Gamma=0$ on $[0, N]$ along trajectory $t\left(\underline{0}, \underline{c}, Q_{0}^{N}\right)$, then $\underline{c}$ can be taken optimally to the origin along the same trajectory for each $\Gamma \geq 0$ on $[0, N]$ and $\underline{c} \varepsilon C_{N}$.

Proof: For $\Gamma=\bar{\Gamma}$ arbitrary, $\underset{N-1}{\operatorname{x}} \varepsilon t\left(\underline{0}, \underline{c}, Q_{0}^{N}\right)$ on $I^{0, N]}$ $v^{0}(N, 0 ; \underline{c}, 0)=\sum_{i=0}^{N-1}\left\|\underline{x}_{i}\right\|_{H^{\prime} H}^{2}=\sum_{i=0}^{N-1}\left\|\underline{x}_{i}\right\|_{H^{\prime} H}^{2}+\left\|\underline{x}_{N}\right\|_{\bar{\Gamma}}^{2} \leq$ $v^{0}(N, \bar{\Gamma} ; \underline{c}, 0)$ by $(I I-2)$ and lemma $a$. Hence $t\left(\underline{o}, \underline{c}, Q_{0}^{N}\right)$ is an optimal trajectory for $\bar{\Gamma}$ on $[0, N]$ and equality holds.

For each $n>N$ let $Q_{0}{ }^{n}$ be the control sequence $Q_{0}{ }^{N}$ augmented by $q_{j}=0 \quad N \leq i \leq n-1$. Then $t\left(\underline{o}, \underline{c}, Q_{0}{ }^{n}\right)$ is a continuation of $t\left(\underline{o}, \underline{c}, Q_{0}^{N}\right)$ for which $\underline{x}_{i}=0, \forall i \quad N+1 \leq i \leq n$. For $\Gamma=\bar{\Gamma}$ arbitrary, $\underline{x} . \varepsilon t\left(\underline{0}, \underline{c}, Q_{0}{ }^{n}\right)$ on $[0, n]$

$$
V^{0}(N, 0 ; \underline{c}, 0)=\sum_{i=0}^{N-1}\left\|\underline{x}_{i}\right\|_{H^{\prime} H}^{2}=\sum_{i=0}^{n-1}\left\|\underline{x}_{i}\right\|_{H^{\prime} H}^{2}+\left\|\underline{x}_{n}\right\|_{\bar{\Gamma}}^{2} \leq V^{0}(n, \bar{\Gamma} ; \underline{c}, 0)
$$

by (II-2) and lemma a. Hence $t\left(\underline{o}, \underline{c}, Q_{0}{ }^{n}\right.$ ) is an optimal trajectory on $[0, n]$ and equality holds. By Theorum $A \quad \underline{c} \varepsilon C_{N}$.

Proof of Theorem B: If: by Lemma b. Conversely, suppose $\underline{c} \varepsilon C_{N}$ for some $N$ finite. Then by Definition 1

$$
V^{0}(N, 0 ; \underline{c}, 0)=V^{0}(N, \Gamma ; \underline{c}, 0) \text { for each } \Gamma \geq 0 \text {. Let } \Gamma_{1}=0 \text { and }
$$
$\Gamma_{2}>0$ arbitrary. Let $\underline{x}^{\prime} \in t_{1}\left(\underline{x}_{N}^{\prime}, \underline{c}, Q_{0}^{N}(1)\right)$ and $\underline{x}^{2} \in t_{2}\left(\underline{x}_{N}^{2}, \underline{c}, Q_{0}^{N}(2)\right)$ denote respectively the optimal trajectories on $[0, N]$ for $\Gamma_{1}$ and $\Gamma_{2}$. Then

$$
\sum_{i=0}^{N-1}\left\|\underline{x}_{i}^{\prime}\right\|_{H^{\prime} H}^{2}=\sum_{i=0}^{N-1}\left\|\underline{x}_{i}^{2}\right\|_{H^{\prime} H}^{2}+\left\|\underline{x}_{-N}^{2}\right\|_{\Gamma_{2}}^{2} .
$$


Assume $\underline{x}_{N}{ }^{2}=\underline{0}$. Then $V^{0}(N, 0 ; \underline{c}, 0)>\sum_{i=0}^{N-1}\left\|\underline{x}_{j}{ }^{2}\right\|_{H^{\prime} H}^{2}$ contradicting optimality for $\Gamma_{7}$. Hence $\underline{x}_{N}^{2}=\underline{0}$ and $t_{2}\left(\underline{0}, \underline{c}, Q_{0}^{N}(2)\right)$ is an optimal trajectory for $\mathrm{T}_{1}=0$ on $[0, N]$.

COROLLARY B.1: $: \underline{c} \varepsilon C_{N}$ for some $N$ finite iff $\exists$ a trajectory of *, $\underline{x} . \varepsilon t\left(0, \underline{c}, Q_{0}^{N}\right)$ on $[0, N]$ which is optimal for each. $r \geq 0$.

Proof: by Theorem B and Lemma b.

The following theorem shows that $C$ is not empty.

THEOREM C: $\phi^{-1} g \in C_{1}$, and for $\forall, n \geq 1 \quad \pi(n, \cdot)_{\phi}^{-1} g=H^{\prime} H_{\phi}^{-1} g$.

Proof: $\pi(n, \cdot) \phi^{-1} g=H^{\prime} H \phi^{-1} g$ follows from (II-3) for $\forall n \geq 1$. Hence $V^{0}(n, \cdot ; \underline{c}, 0)=\left\|\phi^{-1} g\right\|_{H^{\prime} H}^{2}, \forall n \geq 1, \forall \Gamma \geq 0$ for *.

Theorem $C$ contains the result that not only does the quadratic form $\left\|\phi^{-1} g\right\|_{\Pi(n, \cdot)}^{2}$ assume a constant value for $\forall n \geq 1, \forall \Gamma \geq 0$ but that the riccati equation II-3 is constant in direction $\phi^{-1} g$ for $\forall n \geq 1, \forall \Gamma \geq 0$. The next theorem generalizes this result.

THEOREM D: $\quad \underline{c} \in C_{N}$ for some $N$ finite iff for $\forall n, m \geq N$ each $\Gamma_{1} \geq 0$, each $\Gamma_{2} \geq 0 \quad \pi\left(n, \Gamma_{1}\right) \underline{c}=\pi\left(m, \Gamma_{2}\right) \underline{c}$.

Proof: If: by Theorem A. Conversely by Lemma a $v^{0}(k, \Gamma ; \underline{c}, 0) \geq V^{0}(i, 0 ; \underline{c}, 0) \forall k \geq i$, each $\Gamma \geq 0$, each $\underline{c}$. This implies by (II-2) that $\Pi(k, \Gamma)-\pi(i, 0) \geq 0$. If $\underline{c} \varepsilon C_{N}$ then

$$
|| c||_{I I\left(n, \Gamma_{7}\right)-\pi(N, 0)}^{2}=\|\left.\underline{c}\right|_{\Pi\left(m, \Gamma_{2}\right)-\pi(N, 0)} ^{2}=0 \quad \forall n, m \geq N .
$$

Let $\Sigma_{\mathfrak{j}}(\Gamma)=\pi(\boldsymbol{i}, \Gamma)-\pi(N, 0) \quad \boldsymbol{i} \geq N . \quad \Sigma_{\mathfrak{i}}(\cdot) \geq 0$ implies that $\Sigma_{\mathfrak{j}}(\cdot)$ can be factored as $\Sigma_{j}(\cdot)=\Sigma_{j}^{\frac{1}{2}}(\cdot) \Sigma_{j}{ }^{\frac{1}{2}}(\cdot)$. 
Therefore $\left.\quad|| \underline{c}\left\|_{\Sigma_{n}\left(\Gamma_{1}\right)}^{2}=\right\| \underline{c}\right|_{\Sigma_{m}\left(\Gamma_{2}\right)} ^{2}=0$ gives

$$
\Sigma_{n}^{\frac{1}{2}}\left(\Gamma_{1}\right) \underline{c}=\Sigma_{m}^{\frac{1}{2}}\left(\Gamma_{2}\right) \underline{c}=\underline{0} \text {. }
$$

Therefore

$$
\Sigma_{n}\left(\Gamma_{1}\right) \underline{c}=\Sigma_{m}\left(\Gamma_{2}\right) \underline{c}=\underline{0} \forall n, m \geq N
$$

and the result follows.

COROLLARY D.1: $\quad C$ is a linear vector space.

Proof: By Theorem D.

Theorems $B$ and $D$ will be seen to play the principal roles in the analys is that follows.

DEFINITION 4: The $i^{\text {th }}$ moment of system (II- 1 ) is defined to be $H_{\phi}^{i} g=u_{i}, i$ an integer.

Remark: Moments are invariant properties of a system in that they are independent of the coordinate system in which the system is represented.

Remark: $\quad \pi(n, \Gamma)_{\phi}^{-1} g=H^{\prime} u_{-1}$.

Let $R^{i}=\operatorname{span}\left\{\phi^{-1} g, \ldots, \phi^{-i} g\right\}$.

Let $m \leq d$ be the largest positive integer such that $\left\{\phi^{-1} g, \ldots \phi^{-m} g\right\}$ are linearly independent.

Remark: $R^{m}$ is the space of completely controllable states (see [13]).

Lemma c: The vector : $\phi^{-i} g \in R^{m} \forall i, i=0, \pm 1, \ldots$

Proof: see [7].

COROLLARY C.1: Every consecutive "string" of vectors 


$$
\left\{\phi^{i} g, \ldots, \phi^{i+(m-1)} g\right\},
$$

$\forall_{i}, i=0, \pm 1, \ldots$, are linearly independent.

Proof: Obvious.

Lemma d: $C \subset R^{m}$

Proof: If $\underline{c} \varepsilon C$ then by Theorem $B \subseteq$ is a completely controllable state (see [13]). Hence $C \subset R^{m}$.

Lemma e: $\subseteq \varepsilon R^{k}$, $\subseteq \notin R^{k-1}$ cannot be taken to the origin in less than $k$ steps, i.e., on $[0, i], 0 \leq i<k$.

$$
\begin{gathered}
\text { Proof: } \underline{c}=\sum_{i=1}^{k} \phi^{-i} g a_{i} ; a_{k} \neq 0, \quad \text { From (III-2) } \\
\underline{x}_{i}=\phi^{i-k} g a_{k}+\phi^{i-k-1} a_{k-1}+\ldots+\phi^{i-1} g a_{1}+g q_{i-1}+\phi g q_{i-2}+\ldots \\
+\phi^{i-1} g q_{0} .
\end{gathered}
$$

By Corollary c.l and since $a_{k} \neq 0 \quad \underline{x}_{i} \neq \underline{0}$, for $i=0, \ldots, k-1$.

Lemma f: Let $\underline{c} \varepsilon C_{N}$ for some $N$ finite. Let $\underline{x} \varepsilon t\left(\underline{0}, \underline{c}, Q_{0}^{N}\right)$ be an optimal trajectory of $\star$ on $[0, N]$ for $\Gamma=0$ (see Theorem $B$ ). Then $\underline{x}_{i} \in C_{N-i} \forall i, 0 \leq i \leq N-1$.

Proof: By the principle of optimality (see [l]) the trajectories $\underline{x} . \varepsilon t\left(\underline{0}, \underline{x}_{i}, Q_{i}^{N}\right)$ on $[i, N] \forall i, 0 \leq i \leq N-1$. are optimal trajectories of $*$ for $\Gamma=0$. The lemma follows by Theorem $B$ as * is an autonomous system.

Lemma g: Let $\underline{C}_{N} \& C_{N}, \underline{C}_{N} \& C_{N-1}$ for some $N>0$, Let $\underline{x}, \varepsilon t\left(\underline{0}, \underline{c}_{N}, Q_{0}^{N}\right)$ be an optimal trajectory of $*$ on $[0, N]$ for $r=0$ (see Theorem B). Then $\underline{x}_{j} \neq 0$. each $j, 0<j<N$. 
Proof: Suppose $\exists \underline{x}_{j}=\underline{0}, 0<j<N$ and $\underline{x}_{i} \neq \underline{0}, 0 \leq i<j$. Then the trajectory $\underline{\bar{x}}_{\varepsilon} \varepsilon \overline{\mathrm{t}}\left(\underline{\mathrm{o}}, \underline{\mathrm{c}}, \bar{Q}_{0}^{N}\right)$ where $\overline{\mathrm{x}}_{k}=\underline{x}_{k}$ for $0 \leq k \leq j$, $\underline{\bar{x}}_{k}=\underline{0}$ for $j<k \leq N, \quad \bar{q}_{k}=q_{k}$ for $0 \leq k \leq j-1$ and $\bar{q}_{k}=0$, $j \leq k \leq N-1$ is also optimal on $\left[0, N_{j}\right.$ for $r=0$. Since $\underline{x}_{j-1} \neq 0$, $\underline{x}_{j-1}=\phi^{-1} g \alpha$ for some $\alpha=0$ by (II-1). By Theorem $C \underline{x}_{j-1} \varepsilon C_{1}$. Assume that $\underline{x}_{j-\ell} \varepsilon C_{\ell}$ each $\ell, \quad l \leq \ell \leq j$. However, this implies $\underline{x}_{0}=\underline{c}_{N} \varepsilon C_{j}$, where $j<N$. Contradiction : Hence $\exists$ some $k>1$, $j-k \geq 0 \quad 3 \quad \underline{x}_{j-(k-1)} \varepsilon C_{k-1}, \underline{x}_{j-k} \& C_{\ell-1, x_{j-k}} \varepsilon C_{\ell} \quad$ for some $\ell>k$. Let $y=\underline{x}_{j-k}, \quad \underline{w}=\underline{x}_{j-(k-1)}$. Then by (II-1) $\underline{y}=\phi^{-1} \underline{w}-\phi^{-1} g B$ for some $\beta$. By theorems $C$ and $D$ for $\forall n \geq \ell$, each $\Gamma \geq 0$. ** $\Pi(n, \cdot) \underline{y}=\phi^{\prime} \Pi(n-1, \cdot) \underline{w}-\phi^{\prime} \Pi(n-1, \cdot) g\left(g^{\prime} \Pi(n-1, \cdot) g\right)^{\#} g^{\prime} \Pi(n-1, \cdot) \underline{w}+H^{\prime} H y$. Two cases:

(1) $g^{\prime} \Pi(n-1, \cdot) \underline{w}=0, \forall n \geq k$. This means that $\underline{y} \in C_{k}$ since the right side of $\star *$ is constant independent of $\Gamma$ for $\forall n \geq k$. Contradiction: Therefore $\underline{x}_{j} \neq 0$, each $j, 0<j<N$.

(2) $g^{\prime} \Pi(n-1, \cdot)_{\underline{w}}=b \neq 0 \quad \forall n \geq k$. Then ** implies that $\phi^{\prime} \Pi(n-1, \cdot) g\left(g^{\prime} \pi(n-1, \cdot) g\right)^{\#} b$ is constant for $\forall n \geq \ell$ independent of $\Gamma$. Therefore $b^{2}\left(g^{\prime} \pi(n-1, \cdot) g\right)^{\#}$ is constant $\forall n \geq l$, independent of $\Gamma$, i.e., $\mathrm{g}_{\varepsilon} \mathrm{C}_{\ell-1}$. Assume $\ell \geq \mathrm{d}$ otherwise the contradiction is immediate by Lemma $e$ and Theorem B. By Theorem D

$$
\Pi(n, \cdot) g=\phi^{\prime} \Pi(n-1, \cdot) \phi g-\phi^{\prime} \Pi(n-1, \cdot) g\left(g^{\prime} \Pi(n-1, \cdot) g\right)^{\#} g^{\prime} \Pi(n-1, \cdot)_{\phi g}+H^{\prime} H g \text {. }
$$

The term on the left and the two rightmost terms on the right are constant for $\forall \mathrm{n} \geq \ell$, independent of $\Gamma$. Hence $\phi g \in C_{\ell-1}$. It follows inductively that $\phi^{i} g \in C_{\ell-1}, \forall i \geq 0$. But by Lemma $c$ and Corollary $D .1 \quad y \in C_{\ell-1}$. Contradiction : Hence $\underline{x}_{j} \neq 0 \forall j, 0<j<N$. 
The next theorem and its corollaries demonstrate two central properties of $C$ : $C$ is completely characterized by the largest $k \leq m$ such $R^{k} \subset C$ : the riccati equation (II-3) assumes $\Gamma$-independent values in constant directions after a transient period of duration $\leq \mathrm{m}$.

THEOREM E: Let $\underline{c} \varepsilon R^{k}, \underline{c} \& R^{k-1}$ for some $k \leq m$; then $\underline{c} \varepsilon C$ iff $C_{i}=R^{i} \quad i=1, \ldots, k$.

Proof: If: by Corollary D.1 and Definition 2. Conversely, suppose $\underline{c} \varepsilon C$, then $\underline{c} \varepsilon C_{N}$, where $N=\min i{ }^{3} \underline{c} \varepsilon C_{j}$.

By Theorem B and Lemma $\mathrm{N} \geq \mathbf{k}$ and $\exists$ exists an optimal trajectory of $*$ on $[0, N]$, i.e., $\underline{x} .{ }^{\varepsilon}\left(\underline{0}, \underline{c}, Q_{0}^{N}\right)$ for $\Gamma=0$. By Lemma $g$ $\underline{x}_{j} \neq \underline{0}, \quad 0<j<N$. Hence by (II-1) $\quad \underline{x}_{N}=\underline{0}=\phi \underline{x}_{N-1}+g q_{N-1}, q_{N-1} \neq 0$ and $\underline{x}_{N-i}=-\phi^{i} g q_{N-1}-\ldots-\phi^{-1} g q_{N-i} \quad l \leq i \leq N$. By Lemma $f$ $\underline{x}_{N-i} \in C_{j} \quad 1 \leq i \leq N . \quad q_{N-1} \neq 0$ implies that $\underline{x}_{N-i} \quad 1 \leq i \leq k$ are linearly independent vectors. Therefore, for each $\ell, 1 \leq \ell \leq k$ the vectors $\underline{x}_{N-i} \quad 1 \leq i \leq \ell$ span $R^{\ell}$ and by Corollary $D .1 \quad R^{\ell} \subset C_{\ell}$. By Theorem $B$ and Lemma e $R^{\ell}=C_{\ell}$.

COROLLARY E.1: Let $\underline{c} \varepsilon R^{k}$, ç $R^{k-1}$ for some $k \leq m$. If $\underline{c} \in C$ then $R^{k} \subset C_{k}$.

Proof: By Theorem E.

COROLLARY E.2: $\quad C=C_{\ell}=R^{\ell}$ for some $\ell \leq m$.

Proof: By Theorem $C$ and Lemma $d R^{1} \subset C \subset R^{m}$. Let $\ell$ be the largest $k \leq m \quad 3 \quad R^{\ell} \subset C$. Assume $\underline{c} \varepsilon C, \underline{c} \notin R^{l}$. Hence by Lemma $d \underline{c} \varepsilon R^{h}$ some $h, l<h \leq m$. By Corollary E.l $R^{h} \subset C$. Contradiction ! Therefore $C=R^{l}$ and $C=C_{\ell}$ by Theorem $E$. 
The sequence of theorems $F$ - I reveal further properties of the riccati equation (II-3) and provide results equivalent to Theorem E. These will be used to derive an algorithm for determing the size of $C$.

THEOREM $F$ : Let $\underline{c} \in R^{k}, \underline{c} \notin R^{k-1}$ some $k \leq m$. If $\underline{c} \varepsilon C$, then $g^{\prime} \Pi(n, \cdot) \phi^{-i} g=0, \forall \dot{n} \geq i \quad$ each $i, \quad 1 \leq i \leq k-1$.

Proof: By Theorem E $C_{i}=R^{i} i=1, \ldots, k$. Therefore $\phi^{-i} g \varepsilon C_{j}$, $1 \leq i \leq k$. Let $2 \leq i+1 \leq k$, then for $n \geq i+1$ by Theorem $D$

$$
\begin{aligned}
\pi(n, \cdot) \phi^{-(i+1)} g=\phi^{\prime} \Pi(n-1, \cdot)_{\phi}{ }^{-i} g-\phi^{\prime} \Pi(n-1, \cdot) g\left(g^{\prime} \Pi(n-1, \cdot) g\right) \#^{\prime} \Pi(n-1, \cdot)_{\phi}{ }^{-i} g \\
+H^{\prime} H_{\phi}-(i+1) g .
\end{aligned}
$$

If $g^{\prime} \pi(n-1, \cdot) \phi^{-i} g=b_{j} \neq 0$ constant $\forall n-1 \geq i, \quad \Gamma-i n d e p e n d e n t$, then $g \in C_{j}$. By Theorem B, Corollary $c .1$ and Lemma $e$, if $g \in C, g \notin C_{j}$ for $j<m$. But $i<k \leq m$. Contradiction : Hence $b_{i}=0, \forall i \quad i \leq i \leq k-1$.

THEOREM G: If $g^{\prime} \Pi(n, \cdot) \phi^{-i} g=0, \forall n \geq i \quad l \leq i \leq k-1$, then

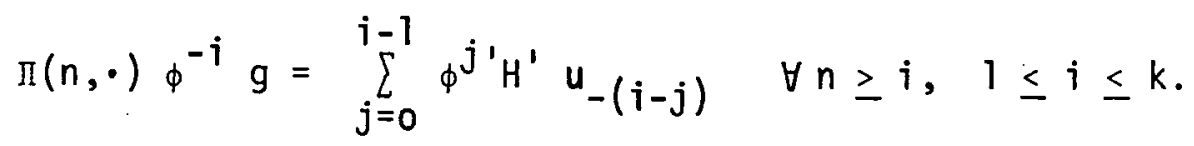

Proof: By Theorem $C \quad \Pi(n, \cdot)_{\phi}^{-1} g=H^{\prime} u_{-1}$. Assume the result holds for $\ell-1<k$. Then for $\ell \leq k$, by (II-3)

$$
\begin{aligned}
\Pi(n, \cdot) \phi^{-l} g & =\phi^{\prime} \Pi(n-1, \Gamma)_{\phi}^{-(l-1)} g+H^{\prime} H_{\phi}^{-l} g \\
& =\sum_{j=0}^{\ell-1} \phi^{j} H^{\prime} u_{-}(l-j)
\end{aligned}
$$

THEOREM H: If $\Pi(n, \cdot)_{\phi}^{-i} g=\sum_{j=0}^{\ell-1} \phi^{j j^{\prime}} H^{\prime} u_{-}(i-j), \forall n \geq i$, $i=1, \ldots, k$, then $c_{i}=R^{i}$.

Proof: by Corollary D.i 
Define for $2 \leq k \leq m$ the vector

$$
w^{k-1}=\left[\begin{array}{c}
\sum_{j=0}^{k-2} u_{j}^{\prime} u_{-(k-j-1)} \\
\vdots \\
u_{0}^{\prime} u_{-2}+u_{1}^{\prime} u_{-1} \\
u_{0}^{\prime} u_{-1}
\end{array}\right]
$$

Note that if $w^{i}=0$ then $w^{i-1}=0$.

$$
\begin{aligned}
& \text { THEOREM I: } W^{k-1}=0, \quad 2 \leq k \leq m \text {, iff } \pi(n, \cdot)_{\phi}^{-i} g \\
& =\sum_{j=0}^{i=1} \phi^{j^{\prime}} H^{\prime} u_{-}(i-j) \quad \forall n \geq i \text {, each } i \quad l \leq i \leq k . \\
& \text { Proof: If: by Theorems } E-H \text { since for } n \geq i g^{\prime} \Pi(n, \cdot) \phi^{-i} g \\
& =\sum_{j=0}^{i-1} u_{j}^{\prime} u_{-(i-j)}=0 \text {, each } i \quad 1 \leq i \leq k-1 ; \quad i . e ., w^{k-1}=0 \text {. } \\
& \text { Conversely, by Theorem } C \text { for } k=2 \quad g^{\prime} \pi(n, \cdot) \phi^{-i} g=u_{0}^{\prime} u_{-1}=0 \text { and }
\end{aligned}
$$$$
\text { by (II-3) } \Pi(n, \cdot) \phi^{-2} g=\phi^{\prime} H^{\prime} H \phi^{-1} g+H^{1} H \phi^{-2} g \text { for } \forall n \geq 2 \text {. }
$$

Assume the result is true for a $j<k$. Then for $j+1 \leq k$ by $($ II -3$)$

$$
\begin{aligned}
\Pi(n, \Gamma)_{\phi}^{-(j+1)} g= & \phi^{\prime} \sum_{\ell=0}^{j-1} \phi^{\ell^{\prime}} H^{\prime} u_{-(j-\ell)} \\
& -\phi \frac{1}{\Pi}(n-1, \Gamma) g\left(g^{\prime} \Pi(n-1, \Gamma) g\right)^{\#} \sum_{\ell=0}^{j-1} u_{\ell}{ }^{\prime} u_{-}(j-\ell)+H^{\prime} u_{-}(j+1) \\
& =\sum_{\ell=0}^{j} \phi^{\ell '} H^{\prime} u_{-}(j+1-\ell) \quad \forall n \geq j+1 \text { since } w^{j}=0
\end{aligned}
$$

and the result follows.

Remark: Theorems E - I are equivalent. 


\section{CHAPTER IV}

\section{MAIN RESULTS}

By Corollary E.2 $C=R^{\ell}$ for some $\ell \leq m$. The next theorem which is the main result, provides a rule for calculating the value of $\ell$.

THEOREM $\mathrm{J}:$ For $2 \leq k<m$. $W^{k-1}=\underline{0} W^{k} \neq \underline{0}$ iff $c=R^{k}$. For $k=m \quad W^{m-1}=\underline{0}$ iff $C=R^{m}$.

Proof: If $C=R^{k}, k \leq m$ then by theorems $E-I W^{k-1}=\underline{0}$. For $k<m$ take $\ell$ arbitrary $k<\ell \leq m$. Suppose $W^{\ell-1}=0$. Then by theorems $I$ and $H \quad R^{\ell} \subset \dot{C}$. Contradiction! Hence $W^{\ell-1} \neq \underline{0}$ each $\ell$ $k<\ell \leq m$. Conversely, if $W^{k-1}=\underline{0}, k \leq m$ then by theorems $I$ and $H$ $R^{k} \subset C$. For $k=m \quad C=R^{m}$ by Lemma $d$. If $k<m$ and $W^{k} \neq \underline{0}$ assume $. \exists \underline{c} \varepsilon C, \underline{c} \& R^{k}$. By Lemma $d, \underline{c} \varepsilon R^{\ell}$ for some $\ell, k<\ell \leq m, \underline{c} R^{\ell-1}$. But theorems $E$ - I imply that $W^{\ell-1}=\underline{0}$, i.e., $W^{k}=\underline{0}$. Contradiction ! Hence $C=R^{k}$.

THEOREM $K$ : If $\underline{c} \varepsilon C$ then $\underline{c}=\sum_{i=1}^{k} \phi^{-i} g a_{i}, a_{k} \neq 0$ some $k \leq m, \quad \underline{c} \in C_{k} \quad$ and

$$
\Pi(n, \cdot) \underline{c}=\sum_{i=1}^{k} \sum_{j=0}^{i-1} \phi^{j^{\prime}} H^{\prime} u_{-(i-j)} a_{i} \quad \forall n \geq k .
$$

Proof: Theorems $E-I$. 


\section{CHAPTER $V$}

\section{FURTHER IMPLICATIONS OF THE RESULTS}

The following theorem describes when the dead-beat control (i.e., the control which minimizes the time to reach the origin) is optimal (see [13]).

THEOREM L: Let $c=\sum_{j=1}^{k} \phi^{-i} g a_{i}, a_{k} \neq 0, k \leq m . \quad \underline{c} \varepsilon c$ iff C can be taken optimally to the origin by for $\forall \Gamma \geq 0$ by the deadbeat control $q_{j}=a_{j+1} \quad 0 \leq i \leq k-1$ on $[0, k]$.

Proof: It follows by Theorem B. Conversely, if $\underline{c} \varepsilon C . \underline{C} \varepsilon C_{k}$, $\underline{c}\left\{C_{k-1}\right.$ by Theorem $E$. Let $\underline{x}, \varepsilon t\left(\underline{o}, \underline{c}, Q_{0}{ }^{k}\right)$. be an optimal trajectory which takes $\underline{c}$ to $\underline{0}$ on $[0, k]$ for $\Gamma=0$ (hence by Lemma $b$ for $\forall \Gamma \geq 0$ ). $\underline{x}_{k}=g\left(a_{k}+q_{k-1}\right)+\phi g\left(a_{k-1}+q_{k-2}\right)+\ldots+\phi^{k-1} g\left(a_{1}+q_{0}\right)$. By Corollary $c .1$ $x_{k}=0$ iff $q_{i}=a_{j+1}, i=0, \ldots, k-1$.

Since a constant direction of the riccati equation (II-3) is $\Gamma$-independent, it is clear by Theorem $D$ that a constant direction is an asymptotically stable equilibrium direction. Also, by Corollary E.2 this equitibrium direction is reached in $m$ or less steps. Once the riccati equation (II-3) reaches the equilibrium point in a constant direction, any further calculation of the value of $P_{n}$ in that direction is redundant. This motivates the following discussion.

DEFINITION 5: Let $C=\mathrm{R}^{\ell}$. Then the dynamic rank of the riccati equation $(I I-3)=d-l$, where $d=$ system dimension. 
Consider that the system (II-I) is in the coordinate system $\Lambda=\left[\phi^{-} g, \ldots, \phi^{-1} g, L\right]=\left[\underline{e}_{j}, \ldots, \underline{e}_{d}\right]$, where $\Lambda$ is a nonsingular $d \times d$ matrix, $i . e .$, the $e_{i}, i=1, \ldots, d$, are a set of basis vectors, and $d-\ell$ is the dynamic rank of the corresponding riccati equation. It is clear from Theorem $B$ and Corollary E.2 that after a transitory period of length $\ell, \pi(n, \Gamma)$ can be partitioned as

$$
\Pi(n, \Gamma)=\left[\begin{array}{ll}
A & B \\
B^{\prime} & D(n, \Gamma)
\end{array}\right], n \geq \& \text { where }
$$

$D(n, \Gamma)$ is $a(d-\ell) \times(d-\ell)$ matrix, and $A$ and $B$ are constant matrices independent of $\Gamma$. From equation (II-3) it follows that $D(n, \ell)$ can be computed recursively in terms of itself, and the constant matrices $A$ and $B$, by means of a $(d-\ell) \times(d-\ell)$ matrix riccati type equation. Note that all $\Gamma$-independent constant singularities are eliminated from the iteration by this reduction since they are a subset of the set of constant directions.

It is of interest to know when the submatrix $D_{n}$ is nonsingular. The following theorem provides a sufficient condition for $D_{n}$ to be nonsingular.

THEOREM M: If $\Gamma>0$ then $D(n, \Gamma)>0 \quad \forall n \geq 0$.

Proof: Suppose $\exists a(d-\ell)$ vector $\underline{c}^{*}, D(n, \Gamma) \underline{c}^{*}=0$ for some $n$. Let $\subseteq$ be the d vector $\underline{c}^{\prime}=\left[0, \ldots, 0, c^{* 1}\right]$. Then for system * with $\Gamma=\Gamma^{*}>0 \quad V^{0}\left(n, \Gamma^{*} ; \underline{c}, 0\right)=\|\underline{c}\|_{\Pi\left(n, \Gamma^{*}\right)}^{2}=\left\|\underline{c}^{*}\right\|_{D\left(n, \Gamma^{*}\right)}^{\prime \prime}$. Let $\underline{x} \in \mathrm{t}\left(\underline{x}_{n}, \underline{c}, Q_{0}{ }^{n}\right)$ be an optimal trajectory of $*$ on $[0, n]$ for $\Gamma^{\star}$. Hence $V^{0}\left(n, \Gamma^{*} ; \underline{c}, 0\right)=\sum_{i=0}^{n-1}\left\|\underline{x}_{i}\right\|_{H^{\prime} H^{2}}^{2}\left\|\underline{x}_{n}\right\|_{\Gamma^{*}}^{2}=0$. Therefore 
$\underline{x}_{n}=0$ and $H \underline{x}_{i}=0 \quad 0 \leq i \leq n-1$. But this trajectory is obviously optimal on $[0, n]$ for $\Gamma=0$. Then by Theorem $B \quad c \varepsilon C$, where $\underline{c}=\sum_{i=\ell+1}^{\ell} \underline{e}_{i} a_{i}$. Contradiction: i.e., $D(n, \Gamma)>0 \quad \forall n \geq 0$. 
CHAPTER VI

APPLICATION TO OPTIMAL LINEAR FILTERING

Consider the system (II-4) - (II-7) under the mapping (II-8).

This is the dual of the optimal control problem * studied in the previous

sections. The results obtained for constant directions of equation (II-3) apply by duality to equation (II-7). For example: define $u_{i}=h_{\psi}{ }^{i} G$, the $i^{\text {th }}$ moment of (II-4), (II-5). Let $\bar{C}$ denote the set of constant directions of (II-7). Let $s^{k}=$ span of $\left\{\psi^{-1} h^{1}, \ldots, \psi^{-k} h^{\prime}\right\}$. Let

$$
y^{k-1}=\left[\begin{array}{lll}
\sum_{j=0}^{k-2} u_{j} u_{-(k-j-1)}^{\prime} \\
u_{0} u_{-2}+u_{1} u_{-1} \\
u_{0} u_{-1}^{\prime}
\end{array}\right]
$$

The dual of Theorem $\mathrm{J}$ is

IHEOREM $N$ : For $2 \leq k<m \quad \gamma^{k-1}=\underline{0}, \gamma^{k} \neq \underline{0}$ iff $\bar{c}=s^{k}$. For $k=m \quad Y^{m-1}=\underline{0}$ iff $\bar{c}=s^{m}$.

Proof: By duality.

The reduction in dynamic rank of the riccati equation (II-3) discussed in the previous chapter applies equally to the riccati equation (II-7) by reason of the duality map (II-8).

It will now be demonstrated that the dual of an optimal trajectory starting at $\underline{c}$ and arriving at the origin in finite time period $[0, k]$ for $\Gamma=0$ is the filter direction $\subseteq$ for which the 
best one-step predictor estimate is a constant linear combination of the last $k$ outputs for $n \geq k-1$. Note that in the proof below the estimate equations hold almost surely.

$\frac{\text { THEOREM 0: }}{-}$ Let $\underline{\lambda}_{k}=\sum_{i=1}^{k} h \psi^{-i} a_{i}$ (a row vector), $a_{k} \neq 0$, $k \leq m . \quad \underline{\lambda}_{k} \varepsilon \cdot \bar{c} \quad$ if and only if

$$
\underline{\lambda}_{k} \cdot \underline{\hat{x}}_{n+1 / n}=\sum_{i=1}^{k} a_{i} z_{n-i+1} \text { a.s., a11 } n \geq k-1 .
$$

(- denotes the vector inner product.)

Proof: If $\lambda_{k} \in \bar{C}$ by (II-6), Theorem $F$ and duality

$\underline{\lambda}_{k} \cdot \hat{\underline{x}}_{n+1 / n}=\underline{\lambda}_{k} \cdot \psi \hat{\underline{x}}_{n / n-1}+a_{1} h \pi(n, \Gamma) h^{\prime}\left(h \pi(n, \Gamma) h^{\prime}\right)^{\#}\left(z_{n}-h \hat{x}_{n / n-1}\right)$, for $n \geq k-1$.

Assume $h \Pi(n, \Gamma) h^{\prime}>0$, then

$$
\begin{aligned}
\underline{\lambda}_{k} \cdot \hat{\underline{x}}_{n+1 / n} & =a_{1} z_{n}+\left(\underline{\lambda}_{k} \psi-h a_{1}\right) \cdot \underline{\underline{x}}_{n / n-1} \\
& =a_{1} z_{n}+\underline{\lambda}_{k-1} \hat{\underline{x}}_{n / n-1}, \text { where } \underline{\lambda}_{k-1}=\sum_{j=1}^{k-1} a_{i+1} h \psi^{-i} .
\end{aligned}
$$

If $h \Pi(n, \Gamma) h^{\prime}=0$ then

$$
\begin{aligned}
\underline{\lambda}_{k} \cdot \underline{\hat{x}}_{n+1 / n}=\underline{\lambda}_{k} \cdot \psi \hat{x}_{n / n-1} & =a_{1} h \underline{\hat{x}}_{n / n-1}+\underline{\lambda}_{k-1} \cdot \underline{\hat{x}}_{n / n-1} \\
& =a_{1} z_{n}+\underline{\lambda}_{k-1} \cdot \underline{\hat{x}}_{n / n-1} .
\end{aligned}
$$

By the dual of Theorem $E, \lambda_{k-1} \varepsilon \bar{C}$ and $i t$ can be shown that the desired implication follows inductively.

Conversely assume that $\underline{\lambda}_{k} \cdot \hat{\underline{x}}_{n+1 / n}=\sum_{j=1}^{k} a_{i} z_{n-i+1}$, all $n \geq k-1$. Note that from equations (II-4) and (II-5) it can be shown inductively 
that

$$
h \psi^{-i} \underline{x}_{n+1}=z_{n-i+1}+h \psi^{-1} G \underline{u}_{n-i+1}+\ldots+h \psi^{-i} G \underline{u}_{n} .
$$

Hence

$$
E\left[{ }_{i=2}^{k} a_{i}\left(h \psi^{-1} G \underline{u}_{n-i+1}+\ldots+h \psi^{-(i-1)} G_{-n-1}\right) \mid z_{j}, j \leq n\right]=0
$$

all $n \geq k-1$, since $E\left[u_{n} \mid z_{j}, j \leq n\right]=0$. By smoothing property of conditional expectation

$$
E\left[\sum_{i=2}^{k} a_{i}\left(n \psi^{-1} G \underline{u}_{n-i+1}+\ldots+h \psi^{-(i-1)} G \underline{u}_{n-1}\right) \mid z_{j}, j \leq n-1\right]=0
$$

al1 $n \geq k-1$ Letting $j=i-1, n=m+1$

$$
E\left[\sum_{j=1}^{k-1} a_{j+1}\left(h \psi^{-1} G \underline{u}_{m+1-j}+\ldots+h \psi^{-j} G \underline{u}_{-m}\right) \mid z_{i}, i \leq m\right]=0,
$$

$$
\text { all } m \geq k-2 \text {. }
$$

Hence

$$
x_{k-1} \cdot \hat{x}_{n+1 / n}=\sum_{i=1}^{k-1} a_{i+1} z_{n-i+1}, \text { all } n \geq k-2 .
$$

Inductively it follows that

$$
\underline{\lambda}_{j} \cdot \hat{\underline{x}}_{n+1 / n}=\sum_{i=1}^{k-j} a_{i+j} z_{n-i+1}, \quad \text { a } 11 \quad n \geq j-1,
$$

where

$$
\lambda_{j}=\sum_{i=1}^{k-j} h \psi^{-i} a_{i+j} .
$$

The $, \frac{\lambda}{k} j, 1 \leq j \leq k$, span the vector space $s^{k}$, and for any $\lambda \in s^{k}$, i.e., $\underline{\lambda}=\sum_{i=1}^{k} h \psi^{-i} b_{i}, \lambda \cdot \underline{x}_{n+1 / n}=\sum_{i=1}^{k} b_{j} z_{n-i+1}, n \geq k$. Since $h_{\psi}^{-i} \varepsilon s^{k}$, each $i, \quad 1 \leq i \leq k$

$$
n \psi^{-i} \underline{x}_{n+1 / n}=h \psi^{-(i-1)} \underline{\hat{x}}_{n / n-1}-h \psi^{-(i-1)} \pi(n, r) h^{\prime}\left(h \pi(n, \Gamma) h^{\prime}\right)^{\#}\left(z_{n}-h \underline{\hat{x}}_{n / n+1}\right) \text {. }
$$


Therefore for $2 \leq i \leq k, n \geq i-1$

$$
z_{n-i+1}=z_{n-i+1}-h \psi^{-(i-1)} \Pi(n, \Gamma) h^{\prime}\left(h \pi(n, \Gamma) h^{\prime}\right)^{\#}\left(z_{n}-h \hat{x}_{n / n-1}\right) \text {. }
$$

Namely,

$$
h \psi^{-(i-1)} \Pi(n, \Gamma) h^{\prime}\left(h \pi(n, \Gamma) h^{\prime}\right)^{\#} h E\left(\underline{x}_{n}-\underline{\hat{x}}_{n / n-1}\right)\left(\underline{x}_{n}-\underline{\hat{x}}_{n / n-1}\right) h^{\prime}=0 \text {, }
$$

or

$$
h \psi^{-(i-1)} \pi(n, \Gamma) h^{\prime}=0,2 \leq i \leq k, n \geq i-1, \text { all } \Gamma \text {. }
$$

Hence by theorems $F-H$ and duality $\lambda_{k} \varepsilon \bar{C}$.

The following corollary enables the immediate characterization of filter directions which are fixed sums of past data.

$$
\begin{aligned}
& \text { COROLLARY } 0.1: \text { If } \gamma^{k-1}=\underline{0} \text {, then } \\
& \qquad \psi^{-i} \underline{\hat{x}}_{n+1 / n}=z_{n-i+1} \text {, all } n \geq i \text {, each } i, 1 \leq i \leq k,
\end{aligned}
$$

a11 $\Gamma \geq 0$.

Proof: By Theorems $\mathrm{N}$ and 0 .

In sum Theorems $\mathrm{N}$ and $\mathrm{O}$ state that one-step prediction in constant directions merely corresponds to a finite length delay line with constant tap gains. One-step prediction in nonconstant directions has the usual dynamic feedback form with varying gains in general.

The results presented above are employed in the following filtering example.

Example:

$$
\underline{x}_{n+1}=\left[\begin{array}{lll}
1 & 1 & 1 \\
1 & 0 & 0 \\
0 & 1 & 0
\end{array}\right] \underline{x}_{n}+\left[\begin{array}{rr}
0 & 1 \\
0 & -1 \\
1 & 0
\end{array}\right] \quad \underline{u}_{n}
$$




$$
z_{n}=\left[\begin{array}{llll}
1 & 1 & 1
\end{array}\right] \quad x_{n}
$$

It can be easily verified that $\mathrm{Y}^{2}=\underline{0}$, hence there are three linearly independent constant directions which in this example means that the riccati equation reaches equilibrium in three iterations. Calculation es tablishes that

and

$$
P_{n}=\left[\begin{array}{rrr}
1 & -1 & 0 \\
-1 & 2 & -1 \\
0 & -1 & 3
\end{array}\right] \text { for all } n \geq 3 \text {, all } \Gamma \text {. }
$$

$$
x_{n+1 / n}=\left[\begin{array}{c}
z_{n} \\
z_{n-1} \\
z_{n-2}
\end{array}\right]
$$




\section{CHAPTER VII}

\section{DISCUSSION}

The concept constant direction of the riccati equation has been defined. It has been shown that as a consequence the dynamic dimension of the riccati equation II- 3 is less than d. An algorithm has been presented which completely characterizes the set of constant direction for the case $s=1$.

It is of interest to note that the data-differencing technique used in [8] has a simple interpretation in terms of constant directions. For the totally augmented state model the covariance function for pure filtering

$$
P_{n / n}=E\left(\underline{x}_{n}-\underline{\hat{x}}_{n / n}\right)\left(\underline{x}_{-}-\underline{\hat{x}}_{n / n}\right)^{\prime}
$$

has singularities in the domain of the (s $X \mathrm{~d})$ observation operator $\mathrm{H}$ where $\mathrm{Z}_{\mathrm{n}}=\mathrm{Hx} \mathrm{x}_{\mathrm{n}}$ i.e., $\mathrm{HP}_{\mathrm{n} / \mathrm{n}}=0$, all $\mathrm{n} \geq 0$. But as the one-step predictor error covariance function $P_{n+1 / n}$ is related to $P_{n / n}$ by $P_{n+1 / n}=\psi P_{n / n} \psi^{\prime}+G G^{\prime}$ (see $\lceil 4]$ ), therefore

$$
\text { - } \quad H \psi^{-1} P_{n+1 / n}=H P_{n / n} \psi^{\prime}+H \psi^{-1} G^{\prime}=H \psi^{-1} G^{\prime} \quad \text { all } n \geq 0 \text {. }
$$


That is, corresponding to the singularities of $P_{n / n}$ in the domain of $\mathrm{H}$ are the constant directions $\mathrm{H} \psi^{-1}$ of $\mathrm{P}_{\mathrm{n} / \mathrm{n}-1}$, for all $\mathrm{n} \geq 1$. This means that prior to any further analysis the dynamic rank of the riccati iteration for $P_{n / n-1}$ is reduced by the dimension of the observations. In addition the dynamics of the one-step predictor estimate equations are reduced by the same factor as

$$
\begin{gathered}
H \varphi^{-1} \hat{x}_{n+1 / n}=H \hat{x}_{n / n-1}+H P_{n} H^{\prime}\left(H P_{n} H^{\prime}\right)^{\#}\left(Z_{n}-H_{n / n-1}\right)=Z_{n} \\
\text { all } n \geq 0
\end{gathered}
$$

by the pseudo-inverse lemma of Kalman in the appendix of reference [11]. Hence only the estimates for the remaining d directions need be found by the usual estimate equations.

In conclusion, data-differencing reduces the computational complexity of the optimal system, however in general it does not give maximum reduction. 


\section{REFERENCES}

1. Bellman R. E., Dynamic Programming, Princeton University Press, (1957)

2. Bryson, A. E., Jr., and Johansen, D. E., "Linear Filtering for Time-Varying Systems Using Measurements Containing Colored Noise", IEEE Transactions, Vol. AC-10, pp. 4-10, January 1965.

3. Bucy, R. S., "Optimum Finite Time Filters for a Special Non-Stationary Class of Inputs", Internal Report B. B. D.600, March 31, 1959, Johns Hopkins Applied Physics Laboratory.

4. Bucy, R. S., "Optimal Filtering with Correlated Noise", J. Math. and Appl. 20 (1967) pp. 1-8.

5. Bucy, R. S., and Joseph, P. D., Filtering for Stochastic Processes with Applications to Guidance, Interscience, New York, (1968).

6. Deyst, J. J., Masters Thesis, Aerospace Department, Massachusetts Institute of Technology, 1964.

7. Gantmacher, F. R., The Theory of Matrices, Vol. I, Chapter III, Chelsea, New York (1960).

8. Henrikson, L. J., Ph. D. Thesis, "Sequentially Correlated Measurement Noise with Applications to Inertial Navigation", Harvard University, 1967.

9. Kalman, R. E., "A New Approach to Linear Filtering and Prediction Problems", J. Basic Engr., Trans. ASME, 82D, pp. 34=45, March 1960.

10. Kalman, R. E., and Bucy, R. S., 'New Results in Lineár Filtering and Prediction Theory", J. Basic Engr., Trans. ASME, 83D, pp. 95-108, March 1961 .

11. Kalman, R. E., "New Methods in Wiener Filtering Theory", Proc. First Symposium on Engineering Applications of Random Function Theory, Purdue University, November 1960, pp. 270-385, Wiley, New York (1963).

12. Kalman, R. E., "Contributions to the The ory of Optimal Contro1", Bol. Soc. Math. Mexicana, pp. 102-119, 1960. 
REFERENCES (Continued)

13. Kalman, R. E., "On the General Theory of Control Systems", Proceedings of the First International Congress of the International Federation of Automatic Control, Vol. I, Moscow, 1960.

14. Kalman, R. E., Englar, T. S., Bucy, R. S., "Fundamenta1 Study of Adaptive Control Systems", Technical Documentary Report No. ASD-TR-61-27, Vol. II, October, 1964.

15. Kalman, R. E., Englar, T. S., "A User's Manual for the Automatic Synthesis Program", NASA CR-475. 


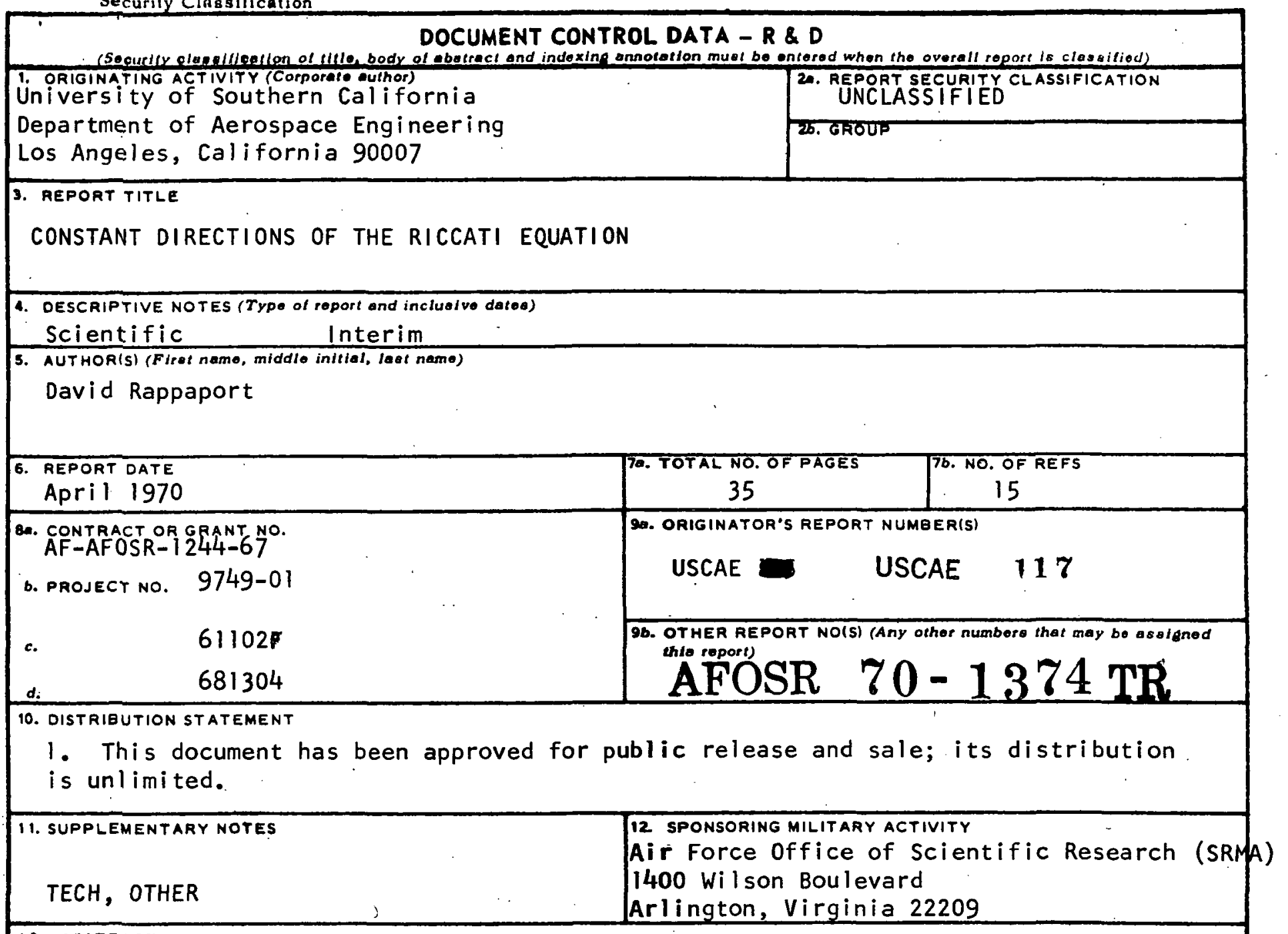

13. AESTRACT

A constant direction of the Riccati equation associated with a class of singular discrete-time optimization problems is defined. The set of constant directions is completely characterized using a control viewpoint. Constant directions are used to reduce the computational complexity of the optimal system. Application to optimal filtering in colored noise is given. 


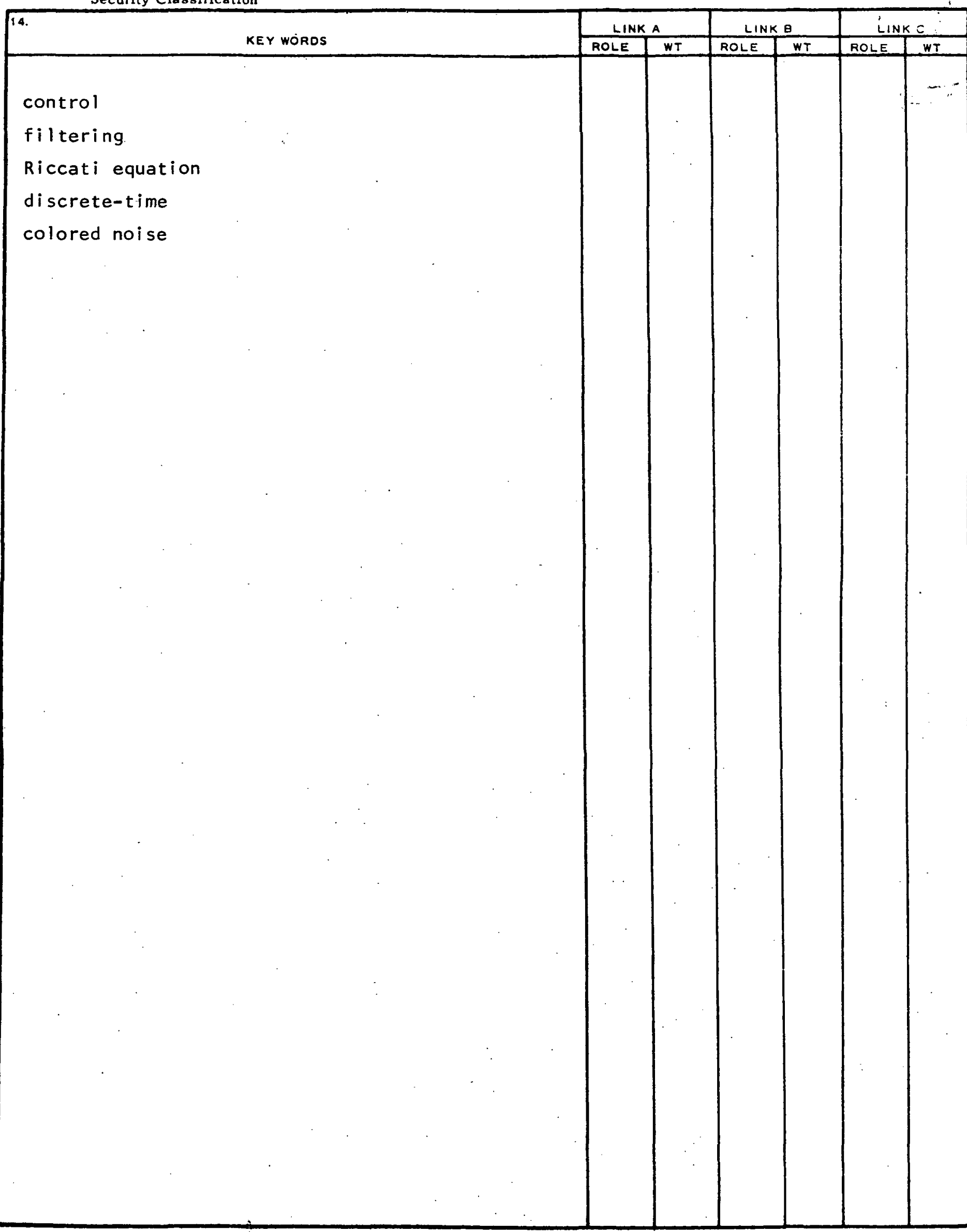

